\title{
GLAST Deficiency in Mice Exacerbates Gap Detection Deficits in a Model of Salicylate-Induced Tinnitus
}

\author{
Hong Yu ${ }^{1,2}$, Kim Vikhe Patil ${ }^{1}$, Chul Han ${ }^{3}$, Brian Fabella ${ }^{4}$, Barbara Canlon ${ }^{1}$, \\ Shinichi Someya ${ }^{3}$ and Christopher R. Cederroth ${ }^{1 *}$ \\ 1 Laboratory of Experimental Audiology, Department of Physiology and Pharmacology, Karolinska Institutet, Stockholm, \\ Sweden, ${ }^{2}$ Department of Otolaryngology, Head and Neck Surgery, First Hospital of JiLin University, Changchun, China, \\ ${ }^{3}$ Department of Aging and Geriatric Research, University of Florida, Gainesville, FL, USA, ${ }^{4}$ Howard Hughes Medical Institute \\ and Laboratory of Sensory Neuroscience, The Rockefeller University, New York, NY, USA
}

OPEN ACCESS

Edited by:

Jianxin Bao,

Northeast Ohio Medical University,

USA

Reviewed by:

Joseph P. Walton,

University of South Florida, USA

Ann E. Hickox,

Decibel Therapeutics, USA

*Correspondence: Christopher R. Cederroth christopher.cederroth@ki.se

Received: 30 March 2016 Accepted: 03 August 2016

Published: 17 August 2016

Citation:

Yu H, Vikhe Patil K, Han C, Fabella B,

Canlon B, Someya S and Cederroth CR (2016) GLAST Deficiency in Mice Exacerbates Gap

Detection Deficits in a Model of

Salicylate-Induced Tinnitus.

Front. Behav. Neurosci. 10:158.

doi: 10.3389/fnbeh.2016.00158
Gap detection or gap pre-pulse inhibition of the acoustic startle (GPIAS) has been successfully used in rat and guinea pig models of tinnitus, yet this system has been proven to have low efficacy in CBA mice, with low basal GPIAS and subtle tinnituslike effects. Here, we tested five mouse strains (CBA, BalbC, CD-1, C57BL/6 and 129sv) for pre-pulse inhibition (PPI) and gap detection with varying interstimulus intervals (ISI) and found that mice from a CBA genetic background had the poorest capacities of suppressing the startle response in the presence of a pre-pulse or a gap. CD-1 mice displayed variable responses throughout all ISI. Interestingly, C57BL/6, 129sv and BalbC showed efficient suppression with either pre-pulses or gaps with shorter ISI. The glutamate aspartate transporter (GLAST) is expressed in support cells from the cochlea and buffers the excess of glutamate. We hypothesized that loss of GLAST function could sensitize the ear to tinnitus-inducing agents, such as salicylate. Using shorter ISI to obtain a greater dynamic range to assess tinnitus-like effects, we found that disruption of gap detection by salicylate was exacerbated across various intensities of a $32-\mathrm{kHz}$ narrow band noise gap carrier in GLAST knockout (KO) mice when compared to their wild-type (WT) littermates. Auditory brainstem responses (ABR) and distortion-product otoacoustic emission (DPOAE) were performed to evaluate the effects on hearing functions. Salicylate caused greater auditory threshold shifts (near $15 \mathrm{~dB}$ ) in GLAST KO mice than in WT mice across all tested frequencies, despite similarly reduced DPOAE. Despite these changes, inhibition using broad-band gap carriers and $32 \mathrm{kHz}$ pre-pulses were not affected. Our study suggests that GLAST deficiency could become a useful experimental model to decipher the mechanisms underlying drug-induced tinnitus. Future studies addressing the neurological correlates of tinnitus in this model could provide additional insights into the mechanisms of tinnitus.

Keywords: hearing loss, salicylate, tinnitus, gap detection, pre-pulse inhibition, startle response, mouse, disease models 


\section{INTRODUCTION}

Tinnitus remains an untreatable condition frequently associated with stress, anxiety or depression (Baguley et al., 2013), and affects $10-15 \%$ of the population. In spite of increasing attention towards the understanding and treatment of tinnitus, experimental efforts in the field remain relatively limited in comparison to the numerous clinical reports (Cederroth et al., 2013). Experimentally, the induction of tinnitus is achieved through noise overexposure or the administration of ototoxic drugs (e.g., salicylate, quinine or cisplatin; Stolzberg et al., 2012; von der Behrens, 2014).

Advances in the field of tinnitus have been made due to the development of behavioral methods to objectively assess the perception of non-existing sounds. Gap pre-pulse inhibition of the acoustic startle reflex (GPIAS), validated in a rat model of tinnitus using the operant conditioning paradigm (Turner et al., 2006; Turner and Parrish, 2008), resembles pre-pulse inhibition of the acoustic startle reflex (PPI or PPIAS), whereby a reduction in the response to an intense stimulus is observed when preceded by a subthreshold intensity pre-pulse (Ison et al., 1973; Graham, 1975). Animal models or humans with schizophrenia or bipolarity disorders have deficits in inhibiting the startle reflex with a pre-pulse as a consequence of a dysfunction in the sensorimotor gating mechanism (Braff et al., 1978; DelPezzo and Hoffman, 1980). In contrast to PPI, which uses a lowintensity pre-pulse to inhibit the startle reflex, GPIAS presents a silent gap embedded in a continuous carrier noise. Despite startle suppression being calculated similarly for both GPIAS and PPI, these use different neural pathways to regulate inhibition. Lesion studies have shown that the auditory cortex regulates GPIAS but not PPI (Bowen et al., 2003). When used in the context of tinnitus, as an animal's tinnitus closely matches the background noise, the startle reflex is less suppressed by the prepulse gap because tinnitus interferes with the optimal inhibition of the startle reflex mediated by the gap. As a consequence, affected animals display greater startle response (meaning less inhibited) than in the absence of tinnitus. The use of GPIAS for the assessment of tinnitus has been supported by additional neuronal correlates of tinnitus such as increased spontaneous firing rates in the dorsal cochlear nucleus (DCN; Li et al., 2013), hyperactivity in the inferior colliculus (Holt et al., 2010) and remapping of the auditory cortex (Engineer et al., 2011).

CBA mice have been conventionally used in auditory research for their excellent hearing abilities. In contrast, C57BL/6, in which mutant strains have been traditionally developed, display age-related hearing loss due to a point mutation on the Cdh23 gene (Ohlemiller and Gagnon, 2004) and thus have been less accepted in the auditory field. However, in the absence of tinnitus, suppression of the startle response with the presence of a gap in narrow band carriers remains highly inefficient in CBA mice (10-50\%; Middleton et al., 2011; Llano et al., 2012; Hickox and Liberman, 2014) when compared to that in rats (40-60\%; Turner et al., 2006; Turner and Parrish, 2008; Engineer et al., 2011; Su et al., 2012; Yi et al., 2016), consequently offering a small dynamic range to distinguish tinnitus-like effects. Thus, improving the basal suppression of gap detection in mice would increase the confidence window for detecting tinnitus. Curiously, mice appear to be resistant to developing tinnitus, as only $20-50 \%$ of the mice that are exposed to noise display behavioral evidence of tinnitus (Middleton et al., 2011; Li et al., 2013), compared to $70-75 \%$ of that in rats (Wang et al., 2009; Zhang et al., 2011; Ruttiger et al., 2013). These observations are consistent with the notion that mice are more resilient than guinea pigs to drug-induced hearing loss by, for instance, aminoglycoside antibiotics or the anti-cancer drug cisplatin (Poirrier et al., 2010). Overall, a smaller number of mice display noise-induced changes in GPIAS than do rats, and this species appears more resistant to drug-induced hearing loss than are guinea pigs.

The glutamate aspartate transporter (GLAST) appears as a potential candidate to explain such differences. GLAST belongs to the family of glutamate transporters that stabilize the extracellular environment and maintain cell-to-cell communication. GLAST, but not GLT1, has been identified in the hearing organ, the cochlea (Jin et al., 2003). GLAST is present in the inner phalangeal cells (IPCs) that surround the sensory inner hair cells (IHCs) and afferent neuron synapse (Ruel et al., 2007), where it pumps back excessive glutamate released by sensory cells (Glowatzki et al., 2006) during noise exposure (Hakuba et al., 2000; Chen et al., 2010). As a consequence, mice lacking GLAST show greater hearing threshold shifts and synaptic damage than do wild-type (WT) mice after noise exposure (Hakuba et al., 2000; Chen et al., 2010). GLAST is hardly detectable in the cochlea of rats or guinea pigs and is abundant in the mouse cochlea (Jin et al., 2003). This is why we hypothesized that GLAST abundance in the murine cochlea could underlie the resilience of mice to inner ear insults by drugs, and potentially tinnitus.

Research on tinnitus would benefit from the use of mice, whose species offers facilitated genetic manipulations for understanding the mechanisms that are related to this auditory disorder. In the present study, we address the limitations of using mice by: (i) increasing the dynamic range of startle suppression in the presence of a gap; and (ii) identifying a protein whose disruption exacerbates gap detection deficits induced by salicylate. By combining these two advances, we propose a new mouse model for investigating tinnitus.

\section{MATERIALS AND METHODS}

\section{Experimental Animals}

Experimental procedures on animals performed at the Karolinska Institutet were in accordance with the guidelines and regulations set out by the University and Stockholm's Norra Djurförsöksetiska Nämnd. Experiments performed at the Rockefeller University were approved by the Institutional Animal Care and Use Committee of The Rockefeller University. GLAST knockout (KO) mice on a C57BL/6 background (Watase et al., 1998) and the other listed strains (from Charles Rivers) were maintained at $19-21^{\circ} \mathrm{C}$ in a $50 \%-50 \%$ light-dark cycle.

GLAST KO mice and their WT littermates were obtained from heterozygous crosses. We observed a non- 
mendelian distribution in the progeny of crosses between heterozygous KO mice ( $n=145)$ : $28 \%$ WT animals, $61 \%$ heterozygous $\mathrm{KO}$ animals, and only $10 \%$ homozygous $\mathrm{KO}$ animals. When crossing male homozygotes with female heterozygotes, we obtained $80 \%$ heterozygous and $20 \%$ homozygous $\mathrm{KO}$ animals among the progeny. In addition, homozygous females appeared highly anxious and displayed pup-killing behavior. Only male mice between 2 and 4 months of age were used in this study. Baseline auditory thresholds of GLAST KO mice measured by auditory brainstem responses $(\mathrm{ABR})$ within this age range were similar to those of WT littermates $(8 \mathrm{kHz}=35.71 \pm 2$; $16 \mathrm{kHz}=19.29 \pm 0.7 ; 32 \mathrm{kHz}=29.64 \pm 1.7 ; \mathrm{KO}:$ $8 \mathrm{kHz}=38 \pm 1.7 ; 16 \mathrm{kHz}=21 \pm 1 ; 32 \mathrm{kHz}=32 \pm 1.33$; $p=0.97$ by a 2 -way ANOVA).

The animals had free access to water and food. They were injected daily with intraperitoneal (i.p.) sodium salicylate (Sigma, S3007) at $300 \mathrm{mg} / \mathrm{kg}$ diluted in $0.9 \% \mathrm{NaCl}$ for three consecutive days. Hearing threshold measures and behavior tests were performed $2 \mathrm{~h}$ after the last injection, as previously described (Guitton et al., 2003).

\section{Auditory Brainstem Responses}

Mice were anesthetized with $80 \mathrm{mg} / \mathrm{kg}$ ketamine and $12 \mathrm{mg} / \mathrm{kg}$ xylazine for measurements of ABR. The positive needle electrode was inserted subdermally at the vertex, the negative electrode was placed beneath the pinna of the left ear, and the ground electrode was located near the tail. ABR were evoked by tone bursts of 8,16 and $32 \mathrm{kHz}$, produced by a closed-field electrostatic speaker connected to a driver (EC-1 and ED-1, Tucker-Davis Technologies). The 5-ms signals were presented 33.3 times per second; their $0.5-\mathrm{ms}$ onsets and offsets were tapered with a squared cosine function. The speaker's audio output was transmitted into the ear through a custom acoustic coupler. Sound pressure levels were measured with a calibrated microphone and preamplifier, connected to a conditioning amplifier (4939-A-011 and 2690A-0S1, Brüel and Kjær). The response was amplified 10,000× and bandpass filtered at $0.3-3 \mathrm{kHz}$ (P55, Natus Neurology Inc.). The amplified response was then digitally sampled at $10-\mu \mathrm{s}$ intervals with a data acquisition device (USB6210, National Instruments), controlled by custom software (LabVIEW 2010, National Instruments). The responses to 1000 bursts were averaged at each intensity level to determine the threshold; the threshold is defined as the lowest level at which a response peak is distinctly and reproducibly present. For each frequency, the sound pressure level was decreased from $100 \mathrm{~dB}$ SPL in $5 \mathrm{~dB}$ steps, until the threshold was reached and confirmed with one replicate measure. Threshold shifts were measured against individual's baseline values.

\section{Distortion-Product Otoacoustic Emissions}

Distortion-product otoacoustic emissions were elicited with an acoustic assembly, consisting of two electrostatic speakers (EC-1, Tucker-Davis Technologies), to generate primary tones and a miniature microphone (EK-23103, Knowles) to measure ear-canal sound pressure. The speakers and the microphone were both calibrated with the calibration microphone described above. The $2 f_{1}-f_{2}$ distortion product was measured with $f_{1}=6-24 \mathrm{kHz}, f_{2} / f_{1}=1.25$ and the stimulus levels $L_{1}=L_{2}=75 \mathrm{~dB}$ SPL. The acoustic signal was amplified by a preamplifier (ER-10B+, Etymotic Research), and the sound pressure measured in the ear canal was digitally sampled at $10-\mu$ s intervals with the data acquisition system described above. Each frequency pair was presented for $1 \mathrm{~s}$. After computing fast Fourier transforms and averaging them over 10 consecutive traces, we determined for each frequency pair the amplitudes of the $2 f_{1}-f_{2}$ distortion product and of the noise floor measured at $\pm 100 \mathrm{~Hz}$ from $2 f_{1}-f_{2}$; this procedure required $17 \mathrm{~s}$ of data acquisition and processing.

\section{Testing of the Interstimulus Interval}

Interstimulus intervals (ISI) were tested using the SR-Lab startle response system from San Diego Instruments as previously described with minor modifications (Lowry et al., 2013). Animals were acclimated to the procedure on day 1 , and were tested for PPI on day 2 and GPIAS on day 5. Background sound level (unfiltered white noise) was $65 \mathrm{~dB}$ SPL for PPI sessions, with prepulses of $75 \mathrm{~dB}$ SPL. For GPIAS, carrier level was $80 \mathrm{~dB}$ SPL and silent gaps (absence of stimulus) went down to the noise floor of the SR-Lab chamber. Startle pulses were presented at $115 \mathrm{~dB}$ SPL. Trials varied pseudo-randomly in their ISI by $10 \mathrm{~ms}$, from 150 to $0 \mathrm{~ms}$. Ten trials per ISI were tested. Inter-trial time interval varied randomly from 8 to $15 \mathrm{~ms}$. Twenty startle-only trials were presented before the session, and five startle-only trials at the end in order to assess habituation. One whole session of PPI or GPIAS lasted $1 \mathrm{~h}$.

\section{Tinnitus Evaluation by the Gap Detection Method}

To test frequency-specific gap detection deficits, we developed a custom-made set-up. Tests were performed in a soundattenuating chamber (ENV-022S, Med Associates, Inc.). whose noise floor was $55 \pm 0.5 \mathrm{~dB}$ SPL. In the case of PPI tests, which were used to verify the normal sensorimotor gating, white noise was generated by a function generator (DS340, Stanford Research Systems) and filtered (Wavetek 852 Dual HI/LO Variable Analog Filter, Butterworth filtered, $48 \mathrm{~dB}$ /octave roll-off) to $1 \mathrm{kHz}$-wide narrowband noise centered at a given frequency or broadband noise $(\mathrm{BBN})$ and presented at a given sound intensity $(60,65,70$, $75,80 \mathrm{~dB}$ SPL) in a silent environment for a duration of $50 \mathrm{~ms}$ through a speaker (NX-6, Power Acoustik) positioned in front of the animal. To startle an animal, a 20-ms white noise startle pulse of $115 \mathrm{~dB}$ SPL was delivered from a second speaker positioned above the animal, $70 \mathrm{~ms}$ after the pre-pulse. Calibration was done using the microphone, preamplifier and conditioning amplifier mentioned above.

For the gap detection tests that were used to evaluate tinnitus perception, the same set-up was used. The gap carrier was filtered as above into a broadband or $1 \mathrm{kHz}$-wide narrowband noise 
centered at a given frequency and presented at a given sound intensity $(60,65,70,75,80 \mathrm{~dB}$ SPL). A relay switch was used to silence the noise for $50 \mathrm{~ms}$, a gap with $0.1 \mathrm{~ms}$ rise and fall times (RT/FT), which was followed $15 \mathrm{~ms}$ later by a 20 -ms white noise startle pulse of $115 \mathrm{~dB}$ SPL. The startle response was captured through an electromagnetic coil, bandpass filtered at 3-100 Hz and amplified $10 \times$ (P55, Natus Neurology Inc.). Gap detection or PPI was quantified as the percentage decrease in the peak amplitude of the startle response, when a warning gap or prepulse preceded the startling noise in comparison to the amplitude when no gap or pre-pulse was present $[(1-$ the ratio $) \times 100]$ (Engineer et al., 2011), using a similar representation as used in PPI studies (greater suppression of the startle reflex closer to $100 \%)$. The more tinnitus fills the gap, the less inhibition of the startle is observed. Only naïve animals were used in this study.

\section{Experimental Procedure for the Screening of the Putative Tinnitus Frequency}

This procedure was performed on a small group of animals $(n=3)$ to identify the putative tinnitus frequency at which a deeper analysis could be performed with a greater number of animals (see below). Here, PPI was not performed in order to avoid excessive habituation to the startle stimulus.

In order to acclimatize the animals to the testing procedure, a 30-min preliminary test was performed on day 1 . On the following day (day 2), the experiment comprised a session of three consecutive blocks.

\section{Block 1}

The first block started with a 5-min acclimatization to silence, followed by 20 startle pulses; each of the pulses was $20 \mathrm{~ms}$ in duration and at $115 \mathrm{~dB}$ SPL. The time between each trial was random and varied between 8 and $12 \mathrm{~s}$.

\section{Block 2}

The second block consisted in testing gap detection at various carrier frequencies (BBN, 8, 10, 12, 16, 20, 24, 32-kHz) of $80 \mathrm{~dB}$ SPL with 20 trials per frequency, with or without silent gaps (50 ms). Each frequency was tested sequentially with 20 trials. Each trial was performed with 20-ms startle pulses and an ISI of $15 \mathrm{~ms}$.

\section{Block 3}

The final block comprised five trials only with startle pulses to be compared with those of the first block to assess habituation. A total of 185 trials was presented in approximately $40 \mathrm{~min}$.

One day after the testing (day 3), animals were treated with salicylate daily for 2 days and tested on the third day of administration (day 5) with the same procedure.

\section{Experimental Procedure for the Validation of the Putative Tinnitus Frequency}

This procedure was performed to validate the findings from the frequency screening performed above. In order to acclimatize the animals to the testing procedure, a 30-min preliminary test was performed on day 1 . On the following day (day 2), the experiment comprised a session of four consecutive blocks.

\section{Block 1}

The first block started with a 5-min acclimation to silence, followed by 20 startle pulses; each of the pulses was $20 \mathrm{~ms}$ in duration and at $115 \mathrm{~dB}$ SPL. The time between each trial was random and between 8 and $12 \mathrm{~s}$.

\section{Block 2}

A second block of stimuli consisted in testing PPI with 20 trials with or without pre-pulses $(50 \mathrm{~ms})$, first consisting of a $\mathrm{BBN}$ of $75 \mathrm{~dB}$ SPL, and then consisting of a $32 \mathrm{kHz}$ centered narrowband noise of successive intensities of $60,65,70,75$ and $80 \mathrm{~dB}$ SPL in a quiet background. Each trial was performed with 20-ms startle pulses and an ISI of $70 \mathrm{~ms}$.

\section{Block 3}

The third block consisted in testing gap detection with 20 trials, with or without silent gaps $(50 \mathrm{~ms})$, first embedded in a broadband carrier noise at $75 \mathrm{~dB}$ SPL and then embedded in a $32-\mathrm{kHz}$ centered narrowband noise of successive intensities of 60 , 65, 70, 75 and $80 \mathrm{~dB}$ SPL. Each trial was performed with $20-\mathrm{ms}$ startle pulses and an ISI of $15 \mathrm{~ms}$.

\section{Block 4}

The final block comprised five trials only with startle pulses to be compared with those of the first block to assess habituation. A total of 265 trials were presented in approximately $55 \mathrm{~min}$.

One day after the testing (day 3), animals were treated with salicylate daily for 2 days and tested on the third day of administration (day 5) with the same procedure.

\section{Quantitative Real Time-PCR}

SybrGreen qRT-PCR assays from cochlear extracts were performed as previously described (Meltser et al., 2014; Vikhe Patil et al., 2015). A mean quantity was calculated from triplicate PCR for each sample, and this quantity was normalized with the geometric mean of the three most stable genes out of six reference genes (tubulin $\beta$, Tubb; glyceraldehyde-3-phosphate dehydrogenase, G3pdh; transferring receptor 1 , Trf1R; Tubulin $\alpha 2$, Tuba2; hypoxanthine phosphoribosyltransferase, HPRT; and Cyclophilin B) selected using the geNorm algorithm as described (Vandesompele et al., 2002). Normalized quantities were averaged for three technical replicates for each data point and represented as mean \pm SD. The highest normalized relative quantity was arbitrarily designated 1.0. Fold changes were calculated from the quotient of means of these normalized quantities and reported as \pm SEM. The primers for eaat 1 used are F: $5^{\prime}$-GGGAAGATGGGGATGCGAG-3' and R: 5'-GCCGAAGCACATGGAGAAG-3'.

\section{Statistical Analysis}

Two-way ANOVA and a Bonferroni post hoc were used for statistical analysis (Prism version 4.0, GraphPad software). Differences were considered significant if $P<0.05$. Animals that 
failed to respond to the startle (any peak-to-peak response above noise floor was considered a startle) or failed to inhibit the startle in the presence of a pre-pulse before salicylate treatment (any decrease in startle amplitude during pre-pulse trials vs. startle only trials) were excluded from the analysis (near 5\%). When performing PPI tests using a pre-pulse of $80 \mathrm{~dB}$ SPL, nearly $10 \%$ of the pre-pulses elicited a startle, which then completely suppressed the startle response. The greater the intensity of the pre-pulse, more efficient was the inhibition of the startle response. As a consequence, trials in which the $80 \mathrm{~dB}$ pre-pulse induced a startle response before the startle pulse $(10 \%$ of the $80 \mathrm{~dB}$ pre-pulse trials) were excluded from the analysis.

\section{RESULTS}

\section{Shorter Interstimulus Interval Improves GPIAS in Specific Mouse Strains}

When developing a custom-made gap detection set-up, we found that the ability of $\mathrm{C} 57 \mathrm{BL} / 6$ mice to inhibit the startle response in the presence of a gap was almost null when using similar settings to those used for PPI (duration of the pre-pulse: $50 \mathrm{~ms}$; ISI: $70 \mathrm{~ms}$; startle duration: $20 \mathrm{~ms}$; startle intensity: $115 \mathrm{~dB}$ ). This particular paradigm only reduced the startle response by $20 \%$ (data not shown). We found that GPIAS was particularly sensitive to modifications of the ISI. We observed that in both C57BL/6 and 129sv mice, unlike for PPI, the shorter the ISI, the greater the inhibition of startle response, achieving up to $80 \%$ in C57BL/6 and 129sv mice (Figures 1B,C). Measures of the startle amplitude before and after each PPIAS or GPIAS session confirmed the lack of habituation during this test (data not shown). While this pattern was present in CBA mice, it did not exceed more than $50 \%$ at the lowest ISI, strongly suggesting that the inhibition of the startle reflex in this strain is not efficient (Figure 1D). This trend was not observed when using other genetic backgrounds such as CD-1 and Balb-C mice (Figures 1E,F). In Balb-C, PPI and GPIAS followed a similar course over varying ISIs, and CD-1 had highly variable responses, suggesting they would require additional acclimatization sessions (as typically performed with rats) to obtain a more robust reflex response. Overall, it is concluded that ISI is a critical parameter for improving gap detection in C57BL/6 and 129sv mice, and that shorter ISIs can be used to increase the basal level of suppression of the startle response in these strains in order to provide greater dynamic range to detect deficits in gap detection.

\section{Salicylate Causes Severe Gap Detection Deficits in GLAST KO Mice}

The basal level of startle suppression in the presence of a gap was further improved using Longenecker and Galazyuk (2012) recommendations by: (i) adjusting speaker non-linearity by calibrating the intensity output for each filtered narrowband noise; (ii) suppressing echo and reverberation by covering the interior walls with sound-insulating foam; and (iii) replacing the acrylic animal restrainer with a non-resonating perforated plastic pipette box. Because noise is effective in inducing tinnitus in nearly $50 \%$ of animals, we used salicylate, which is the most commonly used drug in animal models of tinnitus (Cazals, 2000; von der Behrens, 2014). Salicylate has an advantage over noise in that it has previously been used in humans to induce tinnitus

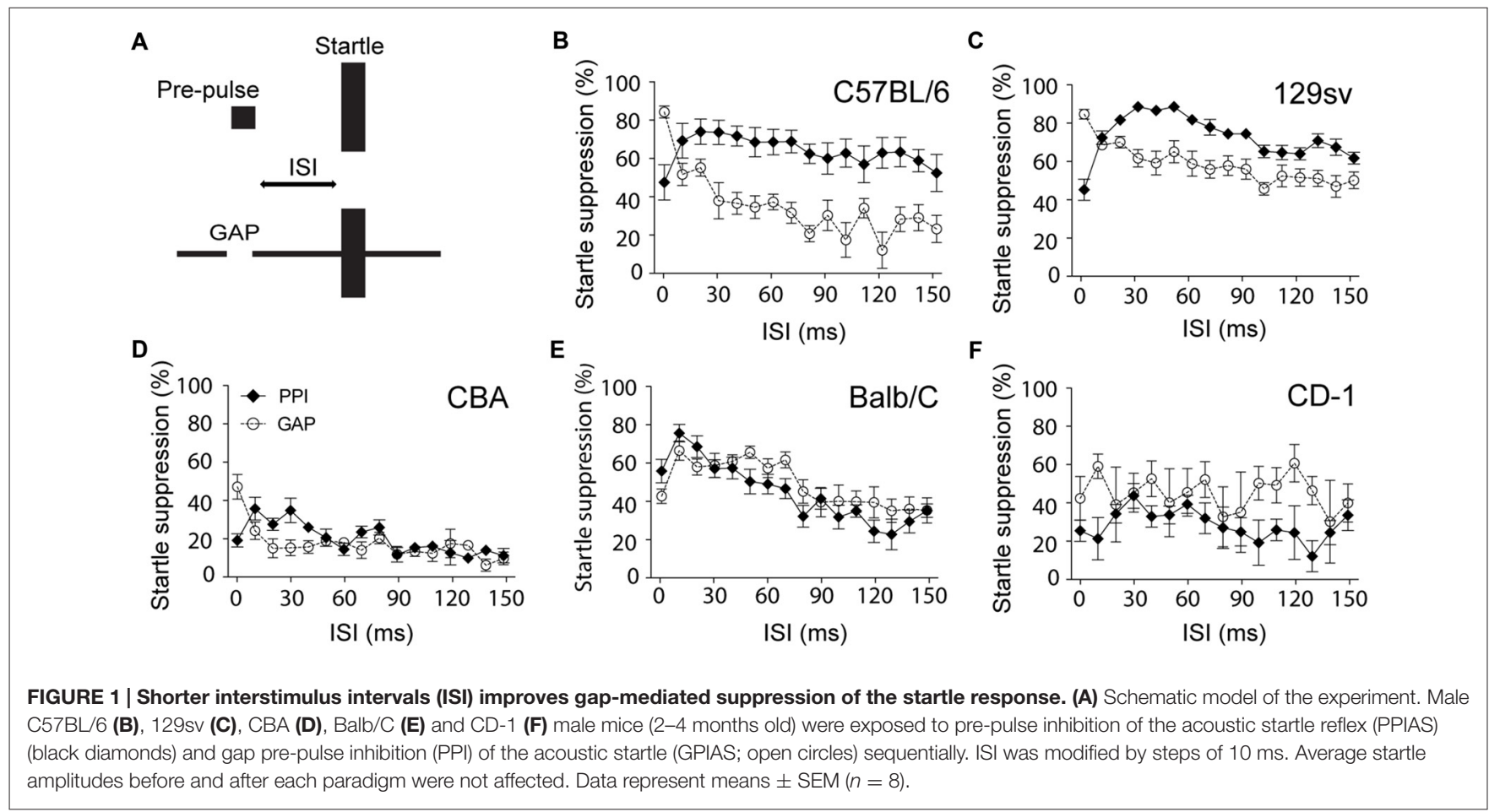


(3.9 g salicylate/day for 5 days; Mongan et al., 1973; McFadden et al., 1984).

We screened various $1-\mathrm{kHz}$ narrowband frequencies (from 8 to $32 \mathrm{kHz})$ in a small group of animals $(n=3)$ to identify the putative tinnitus frequency, meaning the frequency at which GPIAS would be most affected by salicylate treatment according to a previous model $(300 \mathrm{mg} / \mathrm{kg} /$ day for 3 days, Guitton et al., 2003). We used a carrier noise of high intensity ( $80 \mathrm{~dB}$ SPL) to maximize the inhibition of the startle by the gap and identify the frequencies with the greatest changes. This initial screening showed that both WT and KO mice treated with salicylate exhibit greater deficits in sensing the gap at $32 \mathrm{kHz}$ (two-way ANOVA, Genotype and Treatment Factor: $F_{(7,64)}=6.108, p<0.0001$; Frequency Factor: $F_{(3,64)}=11.44, p<0.0001$, data not shown).

We next focused on the $32-\mathrm{kHz}$ narrowband noise to perform both GPIAS, using different carrier intensities, and PPI, using increasing pre-pulse intensities. PPI was used as a control for normal temporal processing or sensory motor gating. Before salicylate administration, WT animals increasingly detected the gap with increasing intensities of a narrowband carrier noise centered at $32 \mathrm{kHz}$, inhibiting their startle reflexes up to $91 \%$ (Figure 2A). After 3 days of salicylate administration, the ability of WT mice to repress their startle reflexes decreased by $25 \%$ at all carrier intensities tested $(p<0.0001$ by a two-way ANOVA with Bonferroni post hoc test, Treatment Factor, $F_{(1,110)}=40.46$, $p<0.0001$; Carrier Intensity Factor, $F_{(4,110)}=35.36, p<0.0001$; Figure 2A). Salicylate did not affect PPI with the exception of the lowest intensity of pre-pulse tested, suggestive of hyperacusis ( $p=0.03$ by a two-way ANOVA with Bonferroni post hoc test, Treatment Factor, $F_{(1,111)}=4.64, p=0.0333$; Pre-pulse Intensity Factor, $F_{(4,111)}=25.40, p<0.0001$; Figure 2B). The overall gap detection deficits, although significant, appeared to be relatively small.

Before salicylate treatment, GLAST KO mice displayed GPIAS as efficiently as WT mice (two-way ANOVA, Genotype Factor: $F_{(1,79)}=0.4351, p=0.5114$; Carrier Intensity Factor: $\left.F_{(4,79)}=57.0, p<0.0001\right)$. However, after salicylate treatment, the ability of GLAST KO mice to detect the gap was severely impaired throughout all carrier intensities tested (two-way ANOVA with Bonferroni post hoc test, Treatment Factor: $F_{(1,89)}=117.5, p<0.0001$; Carrier Intensity Factor: $F_{(4,89)}=16.02, p<0.0001$; Figure 2C). Although PPIAS at $32 \mathrm{kHz}$ appeared to be lower in GLAST KO mice than in WT mice (two-way ANOVA, Genotype Factor: $F_{(1,105)}=17.19, p<0.0001$; Pre-pulse Intensity Factor: $\left.F_{(4,105)}=27.54, p<0.0001\right)$, it was not affected by salicylate administration (two-way ANOVA, Treatment Factor: $F_{(1,105)}=0.2299, p=0.6326$; Pre-pulse Intensity Factor: $F_{(4,105)}=15.72, p<0.0001$, Figure 2D), suggesting that the disruption of gap detection is more likely a tinnitus effect rather than a deficit in temporal processing or in auditory sensitivity (hyperacusislike phenomenon).

To confirm the frequency-specific effects on GPIAS observed after the treatment with salicylate, we used a BBN Gap or pre-pulse (BBN PPI) at $75 \mathrm{~dB}$ SPL, and next verified whether inhibition of the startle response was affected before and

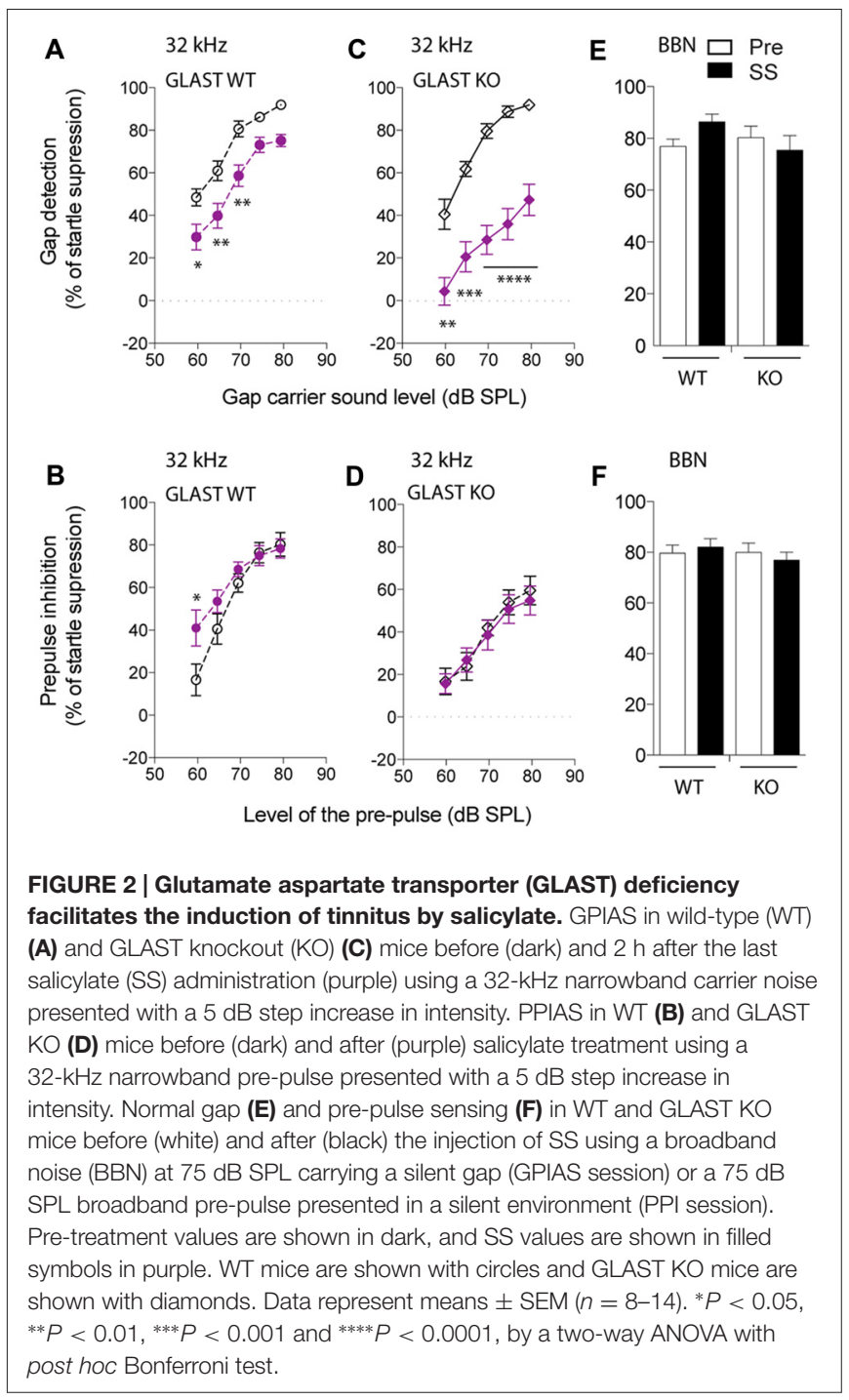

after salicylate administration in both WT and GLAST KO mice. GPIAS in a BBN carrier was equally efficient before or after salicylate treatment in both genotypes (two-way ANOVA, Genotype Factor: $F_{(1,40)}=0.7098, p=0.4045$; Treatment Factor: $F_{(1,40)}=0.2667, p=0.6084$, Figure 2E). Similarly, the efficacy of BBN PPI, which is also used to assess normal temporal processing (Turner et al., 2006; Turner and Parrish, 2008; Middleton et al., 2011; Llano et al., 2012), was identical in WT and mutant animals both before and after the administration of salicylate (twoway ANOVA, Genotype Factor: $F_{(1,46)}=0.4673, p=0.4976$; Treatment Factor: $F_{(1,46)}=0.009994, p=0.9208$, Figure 2F). These control experiments confirmed that BBN PPIAS and GPIAS are not affected by salicylate in both WT and KO mice and support the notion that salicylate causes greater gap detection deficits at $32 \mathrm{kHz}$ in GLAST KO than it does in WT mice.

We also assessed how basal startle amplitudes were affected in WT and GLAST KO mice by salicylate. Salicylate treatment increased the startle amplitude in response to startle pulses alone in KO mice (two-way ANOVA, Genotype Factor: $F_{(1,56)}=37.90$, $p<0.0001$; Treatment Factor: $F_{(1,56)}=17.86, p<0.0001$, 

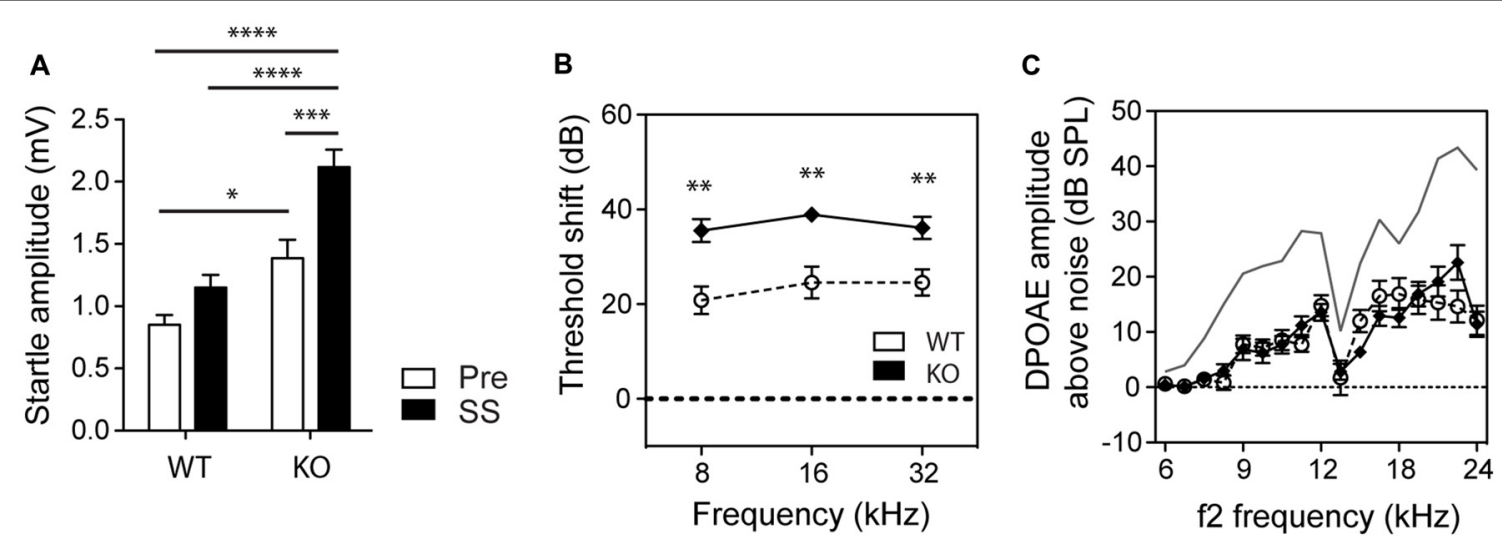

FIGURE 3 | GLAST deficiency sensitizes mice to salicylate-induced hearing loss but without affecting outer hair cell function. (A) GLAST KO mice subjected to startle pulses showed significantly larger startle responses than did WT mice. Salicylate (black, SS) enhanced the startle reflexes of GLAST KO mice. Pre-treatment values are shown in white. Data represent means \pm SEM ( $n=8-14)$. Auditory threshold shifts (B) and distortion-product otoacoustic emissions (DPOAEs C) of GLAST WT (white circles) and homozygous (black diamonds) mice with sodium salicylate at $300 \mathrm{mg} / \mathrm{kg} / \mathrm{day}$ for 3 days, measured $2 \mathrm{~h}$ after the last injection. The uppermost curve (in gray) represents the mean for saline-treated WT control animals analyzed on the same day. Data of the auditory measures represent means $\pm \operatorname{SEM}(n=10-16) .{ }^{*} P<0.05,{ }^{* *} P<0.01$, ${ }^{* * *} P<0.001$ and ${ }^{* * * *} P<0.0001$, by a two-way ANOVA with post hoc Bonferroni test.

Figure 3A). Changes in hearing thresholds could account for differences in gap detection as well as in PPI. It is worth noting that salicylate caused a loss of hearing threshold by $20 \mathrm{~dB}$ across all frequencies (from 8 to $32 \mathrm{kHz}$ ) in WT mice and $35 \mathrm{~dB}$ in GLAST KO mice (two-way ANOVA, Genotype Factor, $F_{(1,57)}=35.03, p<0.0001$; Frequency Factor, $F_{(2,57)}=0.8140, p=0.4482, n=10-16$, Figure 3B) with distortion-product otoacoustic emissions (DPOAEs) reduced by half, and in a similar way in WT and KO mice (Genotype Factor, $F_{(16,340)}=19.05, p=0.9188$; Frequency Factor, $F_{(16,340)}=1.248$, $p<0.0001$, Figure 3C). However, DPOAE measures were only performed at suprathreshold levels, and potential differences at threshold could have occurred. Still, the lack of differences in DPOAEs between WT and KO mice suggests that GLAST does not regulate outer hair cell function; rather GLAST could potentiate the effects of salicylate at the afferent synapse as suggested by the greater threshold shifts in GLAST KO mice. These findings are in agreement with previous work showing that salicylate potentiates glutamate-evoked responses in spiral ganglion neurons ex vivo (Ruel et al., 2008).

Such differences in salicylate-induced threshold shifts between WT and KO should have equally affected the perception of the pre-pulse or the perception of the gap and subsequent PPIAS and GPIAS. However, the findings indicate that this is not the case since PPI remained completely unaffected by salicylate treatment at $32 \mathrm{kHz}$ where gap detection deficits were the greatest (Figures 2B,D). Second, we observed gap sensing deficits in GLAST WT and $\mathrm{KO}$ mice at $32 \mathrm{kHz}$ but not when using a $\mathrm{BBN}$ as a carrier (Figure 2E), although similar hearing threshold shifts have been found across all frequencies tested (Figure 3B). Overall, our results suggest that the gap detection deficits observed here are reminiscent of tinnitus perception in the high-frequency area and are not due to hearing loss or defective temporal processing.

\section{DISCUSSION}

The present study shows that suppression of the startle response in the presence of a gap can be improved in C57BL/6 and $129 \mathrm{sv}$ mice by shortening the ISI and that constitutive loss of glutamate transporter function likely exacerbates the tinnitusinducing effects of salicylate.

In some strains of mice, the efficacy of the startle suppression by the gap requires shorter ISI than with PPI. With a 15-ms ISI, gap detection suppressed the startle response by nearly $80 \%$ in C57BL/6 mice. Our findings contrast with those in the previous studies in rats by Ison and Bowen (2000), in which a biphasic response in the ability of the gap to suppress the startle was observed when varying the ISI . It is likely that these differences are related to species differences. Our results also underline the importance of the genetic background when performing GPIAS experiments. Previous studies have shown that PPI is highly influenced by genetic background and that the C57BL/6 strain displays rather efficient abilities to suppress the startle response in the presence of a pre-pulse (Willott et al., 2003). It would thus be interesting to evaluate how GPIAS is affected by varying ISIs in humans and how genetic background (e.g., different ethnicities) affects the efficacy of GPIAS.

In spite of the successful use of gap detection to identify physiological and molecular pathways involved in tinnitus (Engineer et al., 2011; Li et al., 2013, 2015; Kalappa et al., 2014, 2015), there has been a lot of debate regarding the use of gap detection for tinnitus evaluation (Campolo et al., 2013; Fournier and Hébert, 2013; Boyen et al., 2015; Galazyuk and Hébert, 2015). First, the low level of startle suppression in the presence of a gap reported in tinnitus studies that have used CBA mice leaves little margin to identify gap detection deficits and infer the presence of tinnitus (Longenecker and Galazyuk, 2011; Hickox and Liberman, 2014; Longenecker et al., 2014). Methods to discriminate between tinnitus and non-tinnitus 
animals have proven useful (Li et al., 2013, 2015) but an increase in the basal suppression level of the startle response offers a greater dynamic range for identifying tinnitus with greater confidence. Second, Hickox and Liberman (2014) performed a tinnitus study in which they adjusted the gap such that some of the trials were done with a gap closer to the startle stimulus (ISI $=0 \mathrm{~ms}$ ) and some were done with a gap presented at greater lead times (ISI $=70 \mathrm{~ms}$ ). Consistent with our findings (Figure 1), differences in gap detection between the two ISI conditions were found (being less efficient with greater lead times), however the authors interpreted that tinnitus "was not filling" the gap, since deficits in GPIAS were not observed at both ISIs (Hickox and Liberman, 2014). We believe the experimental conditions used were not optimal for inferring the presence of tinnitus as they used CBA mice, whose basal level of startle suppression in the presence of the gap was inefficient and even more variable with greater ISI. In addition, it has been shown that scopolamine, a muscarinic receptor blocker that disrupts cholinergic function in the brain, affects gap detection when the ISI is larger and not when the gap is closer to the startle stimulus (Ison and Bowen, 2000), meaning that different mechanisms operate GPIAS depending on the ISI. As a consequence, since GPIAS is a reflex response that relies on temporal processing and not on a conscious percept (meaning tinnitus does not fill the gap but interferes with the reflex response per se), gap detection deficits caused by tinnitus cannot be expected to be equally efficient at various lead times. The mechanism with which salicylate interferes solely with GPIAS and not PPI is not well understood, however it has been shown that gap detection operates in the auditory cortex, which is not the case of PPI (Bowen et al., 2003). Recent optogenetic studies in mice have shown that cortical inhibition is important in controlling perceptual gap detection (Weible et al., 2014). Since salicylate has been shown to alter the activity in the auditory cortex (Wang et al., 2006; Sun et al., 2009), we postulate that salicylate alters perceptual gap detection at the level of the auditory cortex. Additional behavioral methods in mice would be needed to confirm that the GPIAS deficits observed here correlate with a tinnitus percept.

Previous studies have shown that the ability to suppress the startle response in the presence of a gap improves with experience or with repeated pre-acclimatization sessions (Crofton et al., 1990; Ison and Bowen, 2000), and can reach 50\% of startle suppression in CBA mice (Ison et al., 2002). The same applies to PPI (Plappert et al., 2006). We believe that the CBA strain, which is commonly used in the auditory field because of the well-preserved hearing, is not appropriate for PPIAS and GPIAS studies, unless longer acclimatization sessions are performed to achieve efficient gap processing. Instead, C57BL/6 or C57BL/ $6 \times 129 \mathrm{sv}$ mixed mice (typical of most mouse mutant models available) would be most appropriate at an age when PPIAS/GPIAS responses are maximized and hearing deficits not yet detectable (between 3 and 4 months of age). Longenecker and Galazyuk (2012) also improved gap detection by decreasing the startle stimulus intensity to non-saturating levels (around $105 \mathrm{~dB}$ SPL), which has not been implemented in the current experiment. The differences we observe may also be due to differences in sound quality. With the exception of the initial study by Turner and Parrish (2008); Hickox and Liberman (2014), who describe a $48 \mathrm{~dB} /$ octave roll-off in their sound filtering like the one used in the present report, none of the other studies that used GPIAS describe the frequency filtering slope of their narrowband noises. Typically, when the shape of the filter is too narrow (e.g., when the slope of the filter is steep), perceptual artifacts are generated and could interfere with the ability of tinnitus to disrupt GPIAS. These artifacts decrease when using a $48 \mathrm{~dB} /$ octave roll-off, and could contribute to improved "detectability" of tinnitus. How the contour of the narrowband filtering affects the ability of tinnitus to interfere with GPIAS remains to be addressed. We believe that the appropriate selection of background strain, startle impulse level and ISI can provide robust PPI and gap detection responses when combined with adequate acclimatization sessions. Such parameters should be taken into account when testing gap detection for the assessment of tinnitus in humans.

The identification of GPIAS deficits in the $32-\mathrm{kHz}$ frequency region when using salicylate in GLAST KO mice contrasts with previous research. A recent review article by Galazyuk and Hébert (2015) summarizes the results obtained with different tinnitus models using GPIAS. It appears that the generation of tinnitus by salicylate varies in terms of frequency depending on species and strains (i.e., Wistar rats get broader-range tinnitus, Brown Norways display tinnitus at 10, 12 and $24 \mathrm{kHz}$, and Sprague Dawleys at $16 \mathrm{kHz}$ ). The overall conclusion is that tinnitus does not seem to be focal and its frequency depends on the strain used. It is possible that we may have missed some frequencies in our screening that would have been otherwise revealed using lower carrier sound intensities and a greater number of animals throughout the procedure. Conversely, the protocols using GPIAS to assess tinnitus are very diverse and none of the salicylate studies (with the exception of the article by Turner and Parrish, 2008) have tested potential GPIAS deficits at $32 \mathrm{kHz}$ (Galazyuk and Hébert, 2015). It is thus hard to determine whether our findings are specific to our model, or whether salicylate-induced tinnitus typically triggers highfrequency tinnitus, or simply whether the deficits in GPIAS are truly triggered by tinnitus per se and do not result from confounding effects of hearing loss. The control experiments we have performed rule out the potential bias of high-frequency hearing loss in the disrupted GPIAS observed in GLAST KO mice since: (i) auditory thresholds were equally affected by salicylate in WT and KO mice at all frequencies; (ii) salicylate did not affect GPIAS using a BBN carrier sound; (iii) basal auditory thresholds were normal and equivalent in both $\mathrm{WT}$ and $\mathrm{KO}$ mice at the time of the test; and (iv) salicylate treatment did not disrupt PPI in either WT or KO mice at $32 \mathrm{khZ}$, which otherwise would have been altered if hearing loss had contributed to the lower GPIAS at $32 \mathrm{kHz}$ found in GLAST KO mice treated with salicylate. However, we do not rule out the possibility that in spite of the significant changes in GPIAS (in either genotype), the lack of changes in PPI upon salicylate administration could arise from salicylate-induced loudness recruitment, which may maintain suprathreshold PPI behavior. 
Our study proposes the first gene potentially involved in tinnitus. GLASTs are mainly present in astrocytes in the central nervous system, buffering the excess of glutamate released at the synaptic cleft, converting it into glutamine, which is then transported back to pre-synaptic terminals to be recycled to glutamate. Two major glutamate transporters, namely GLAST (predominant in the cortex and hippocampus) and the glial GLutamate Transporter GLT1 (predominant in the cerebellum), are responsible for more than $80 \%$ of the glutamate uptake in the brain. Loss of glutamate transporters with subsequently uncontrolled extracellular glutamate levels has been associated with neurotoxic effects during seizures, amyotrophic lateral sclerosis, epilepsy and now, possibly, tinnitus. Although species comparison in gene expression levels is difficult to evaluate, given the broad changes in endogenous normalizing components (e.g., ubiquitously expressed genes), it appears likely that the resistance of mice to auditory insults could result, at least in part, from the higher expression of cochlear GLAST. On the other hand, a low abundance of cochlear GLAST would predict higher sensitivity to noise and drug-induced tinnitus. Consistent with this notion, we found that GLAST mRNA abundance was greater in CBA mouse cochleae than in those from CD-1 mice (CBA: $1.02 \pm 0.06$ relative expression level; CD-1: $0.76 \pm 0.04 ; p=0.008$ by an unpaired two-tailed $t$-test, $n=5$ per strain), which correlates with these strains' known auditory sensitivity and development of age-related hearing loss. In this regard, Shimizu et al. (2005) found that GLAST KO mice are more vulnerable to kanamycin ototoxicity. Ongoing data collection in our laboratory indicates that this is also the case for cisplatin (unpublished observations). In humans, whose susceptibility to ototoxic medications and noise varies from one individual to another, it remains unknown how cochlear GLAST levels correlate with tinnitus predisposition. A recent study failed to detect GLAST protein in support cells from the human cochlea (Ahmed et al., 2013), suggesting that the human cochlea expresses very low levels in GLAST at the afferent synapse. As a consequence, we predict that humans would show less glutamate-buffering capacity and thus greater vulnerability to noise and ototoxic medications. Genetic analyses of the human homolog of GLAST (EAAT1) in subjects with and without tinnitus could bring new knowledge about the mechanisms underlying the vulnerability and resilience to tinnitus.

It is rather widely accepted that peripherally generated tinnitus arises from a lack of cochlear output, rather than an increase in cochlear output. There are clear cochlear differences between salicylate and noise insults whereby spontaneous activity of the auditory nerve (AN) is increased after salicylate but decreased after noise (Eggermont and Roberts, 2004). However, most measures in noise-traumatized animals are performed after noise exposure and not during noise exposure. Thus, we would predict an early phase of increased AN activity during noise exposure (Searchfield et al., 2004) and a decrease in AN activity resulting from permanent synaptic damage. In contrast to noise, measures on salicylate-treated animals are performed shortly after the administration of salicylate-presumably when salicylate bioavailability peaks in the cochlea. Although there are to our knowledge no studies that report salicylate bioavailability in the cochlea after intraperitoneal injections, a peak is observed in other tissues after 30-60 min, with blood clearance after $8 \mathrm{~h}$ (Sturman et al., 1968). As salicylate potentiates glutamate-evoked responses in primary afferent neurons (Ruel et al., 2008), and lack of GLAST results in increased excess glutamate at the IHCcochlear nerve synapse (Hakuba et al., 2000; Glowatzki et al., 2006), the overall cochlear output in GLAST KO mice would be expected to increase before salicylate is cleared out. Whether repeated administration of salicylate would cause permanent damage is unclear, but a recent rat model of chronic salicylateadministration leads tinnitus (Yi et al., 2016) and could prove useful to investigate whether more permanent damages would occur at the synapse, and reconcile salicylate-induced tinnitus with the decreased cochlear output theory.

The tinnitus effects observed here could also underlie central actions of salicylate and/or loss of GLAST function in the brain. Indeed, systemic administration of salicylate could activate non-auditory structures (Stolzberg et al., 2012; Chen et al., 2015) translating into broader central impacts than those happening after noise exposure (Holt et al., 2010). However, GLAST is predominant in the cortex and the hippocampus, and whether it is present in structures of the auditory pathway is unknown. The higher baseline startle amplitudes observed in GLAST KO mice could reflect hyperacusis. These findings are also consistent with the known higher anxiety levels in this model (Karlsson et al., 2009): increased locomotor activity in the open field, decreased sociability and social novelty preference, and poor nesting behavior. In addition, (Karlsson et al., 2009) found that pairwise discrimination learning is also affected in GLAST KO mice. The startle responses we obtain are however inconsistent with Karlsson et al.'s (2009) findings, which were not controlled-as we did with $\mathrm{ABR}$ - for hearing levels (as acknowledged by the authors), known to be affected at 6 months of age in GLAST KO mice (Hakuba et al., 2000). Importantly, unlike the auditory field, which uses startle response as an indicator of hearing abilities, all biological psychiatry textbooks mention the use of the startle response as a gauge of fear or anxiety. For instance, people with post-traumatic stress disorder have greater startle responses (Grillon et al., 1998). After an animal has learned to associate a specific stimulus with fear, such as light being paired with shocks, greater startle response is observed after presenting a fear signal just before the startle stimulus. Conversely, signals associated with pleasure decrease the startle amplitude response (Schmid et al., 1995). Fear or pleasure signals fail to modulate the startle response in amygdala-lesioned animals, showing that the amygdala is involved in the startle response system (Hitchcock and Davis, 1991). We thus believe that the increased startle response seen in GLAST KO mice in the presence of salicylate is an indicator of amygdalamediated effects. The recent evidence linking tinnitus with the amygdala suggests that central-GLAST deficiency in the amygdala could contribute to the increased tinnitus severity observed in GLAST KO mice. Nonetheless, Karlsson et al. (2009) also found that PPI was not affected in GLAST KO mice and hence reasoned that these mice display some of the 
multiple and complex symptoms belonging to schizophrenia (e.g., GLAST KO mice would belong to a subgroup of schizophrenia of lesser severity). Salicylate, which is known to be anxiogenic in high doses (Puel and Guitton, 2007; Guitton, 2009), exacerbated this anxiety effect by increasing startle amplitude responses in $\mathrm{KO}$ animals (Figure 3A), something that we were able to qualitatively observe when handling the animals. It is thus possible that the higher basal anxiety levels of GLAST KO mice facilitated the tinnitus-inducing effects of salicylate, thereby increasing tinnitus intensity. Specific deletion of GLAST either in the brain or in the ear should enable discrimination of the contribution of all of these factors.

\section{CONCLUSION}

Our results suggest a potential role for GLAST in the vulnerability to salicylate-induced tinnitus. Given the magnitude of the disruption in gap detection observed in GLAST KO mice treated with salicylate, we propose that GLAST deficiency may serve as a useful model to distinguish more subtle, yet unidentified mechanisms on how tinnitus is triggered and maintained. Finally, optimizing parameters in gap detection in humans may uncover a potential use of this technology in the objective diagnosis of tinnitus (Galazyuk and Hébert, 2015).

\section{AUTHOR CONTRIBUTIONS}

$\mathrm{HY}, \mathrm{KVP}, \mathrm{CH}, \mathrm{BF}$ and CRC carried out the experiments; HY, KVP and CRC analyzed the results; CRC designed and directed the research; $\mathrm{BC}, \mathrm{SS}$ and CRC discussed the results and wrote the manuscript; all authors reviewed the manuscript.

\section{REFERENCES}

Ahmed, S., Vorasubin, N., Lopez, I. A., Hosokawa, S., Ishiyama, G., and Ishiyama, A. (2013). The expression of glutamate aspartate transporter (GLAST) within the human cochlea and its distribution in various patient populations. Brain Res. 1529, 134-142. doi: 10.1016/j.brainres.2013. 06.040

Baguley, D., McFerran, D., and Hall, D. (2013). Tinnitus. Lancet 382, 1600-1607. doi: 10.1016/S0140-6736(13)60142-7

Bowen, G. P., Lin, D., Taylor, M. K., and Ison, J. R. (2003). Auditory cortex lesions in the rat impair both temporal acuity and noise increment thresholds, revealing a common neural substrate. Cereb. Cortex 13, 815-822. doi: 10 . 1093/cercor/13.8.815

Boyen, K., Başkent, D., and van Dijk, P. (2015). The gap detection test: can it be used to diagnose tinnitus? Ear Hear. 36, e138-e145. doi: 10.1097/aud. 0000000000000156

Braff, D., Stone, C., Callaway, E., Geyer, M., Glick, I., and Bali, L. (1978). Prestimulus effects on human startle reflex in normals and schizophrenics. Psychophysiology 15, 339-343. doi: 10.1111/j.1469-8986.1978.tb01390.x

Campolo, J., Lobarinas, E., and Salvi, R. (2013). Does tinnitus "fill in" the silent gaps? Noise Health 15, 398-405. doi: 10.4103/1463-1741.121232

Cazals, Y. (2000). Auditory sensori-neural alterations induced by salicylate. Prog. Neurobiol. 62, 583-631. doi: 10.1016/s0301-0082(00)00027-7

Cederroth, C. R., Canlon, B., and Langguth, B. (2013). Hearing loss and tinnitus-are funders and industry listening? Nat. Biotechnol. 31, 972-974. doi: $10.1038 /$ nbt.2736

\section{FUNDING}

CRC was a recipient of postdoctoral fellowships from the Swiss National Science Foundation (SNF; n PBGEP3-125837), the Schweizerischen Stiftung für medizinisch-biologische Stipendien (SSMBS; $\mathrm{n}^{\circ}$ PASMP3-136979), the Nicholson Fund and the Wenner Gren Foundation, and has received funding from Vetenskapsrådet, Lars Hiertas Minne, Magnus Bergvalls Stiftelserna, Loo och Hans Ostermans, Tysta Skolan, Karolinska Institutet and an independent research program funded under the Biomedicine and Molecular Biosciences European Cooperation in Science and Technology (COST) Action framework (TINNET BM1306). BC received funding from Karolinska Institutet, Vetenskapsrådet, Tysta Skola, Hörselskadades Riksförbund, FORTE and AFA Försäkring. SS is funded by the American Federation for Aging Research, by National Institutes of Health grant DC011840, and by a Claude D. Pepper Older Americans Independence Center Junior Scholar Award.

\section{ACKNOWLEDGMENTS}

We would like to thank the Hudspeth laboratory for their continuous and generous support, Y. Castellanos for technical assistance, S. Rasmussen for dedicated veterinary advice and J. Dyrhfjeld-Johnsen for his insightful comments on the manuscript. With the approval of K. Tanaka, V. P. Sarthy kindly provided the GLAST KO mice. We are grateful to J. G. Turner and T. Tzounopoulos for advice concerning the gap detection method; M. Ravitch, I. J. Stefanov-Wagner and M. C. Liberman for suggestions about the apparatus for recording $\mathrm{ABR}$ and distortion-product otoacoustic emissions, and for providing an acoustic coupler and calibration software.

Chen, Z., Kujawa, S. G., and Sewell, W. F. (2010). Functional roles of high-affinity glutamate transporters in cochlear afferent synaptic transmission in the mouse. J. Neurophysiol. 103, 2581-2586. doi: 10.1152/jn.00018.2010

Chen, Y. C., Li, X., Liu, L., Wang, J., Lu, C. Q., Yang, M., et al. (2015). Tinnitus and hyperacusis involve hyperactivity and enhanced connectivity in auditorylimbic-arousal-cerebellar network. Elife 4:e06576. doi: 10.7554/elife.06576

Crofton, K. M., Dean, K. F., Sheets, L. P., and Peele, D. B. (1990). Evidence for an involvement of associative conditioning in reflex modification of the acoustic startle response with gaps in background noise. Psychobiology 18, 467-474.

DelPezzo, E. M., and Hoffman, H. S. (1980). Attentional factors in the inhibition of a reflex by a visual stimulus. Science 210, 673-674. doi: 10.1126/science.7433993

Eggermont, J. J., and Roberts, L. E. (2004). The neuroscience of tinnitus. Trends Neurosci. 27, 676-682. doi: 10.1016/j.tins.2004.08.010

Engineer, N. D., Riley, J. R., Seale, J. D., Vrana, W. A., Shetake, J. A., Sudanagunta, S. P., et al. (2011). Reversing pathological neural activity using targeted plasticity. Nature 470, 101-104. doi: 10.1038/nature09656

Fournier, P., and Hébert, S. (2013). Gap detection deficits in humans with tinnitus as assessed with the acoustic startle paradigm: does tinnitus fill in the gap? Hear. Res. 295, 16-23. doi: 10.1016/j.heares.2012.05.011

Galazyuk, A., and Hébert, S. (2015). Gap-Prepulse Inhibition of the Acoustic Startle Reflex (GPIAS) for tinnitus assessment: current status and future directions. Front. Neurol. 6:88. doi: 10.3389/fneur.2015.00088

Glowatzki, E., Cheng, N., Hiel, H., Yi, E., Tanaka, K., Ellis-Davies, G. C., et al. (2006). The glutamate-aspartate transporter GLAST mediates glutamate uptake at inner hair cell afferent synapses in the mammalian cochlea. J. Neurosci. 26, 7659-7664. doi: 10.1523/JNEUROSCI.1545-06.2006 
Graham, F. K. (1975). Presidential Address, 1974. The more or less startling effects of weak prestimulation. Psychophysiology 12, 238-248. doi: 10.1111/j.14698986.1975.tb01284.x

Grillon, C., Morgan, C. A. III, Davis, M., and Southwick, S. M. (1998). Effects of experimental context and explicit threat cues on acoustic startle in Vietnam veterans with posttraumatic stress disorder. Biol. Psychiatry 44, 1027-1036. doi: 10.1016/s0006-3223(98)00034-1

Guitton, M. J. (2009). Tinnitus-provoking salicylate treatment triggers social impairments in mice. J. Psychosom. Res. 67, 273-276. doi: 10.1016/j.jpsychores. 2008.10.017

Guitton, M. J., Caston, J., Ruel, J., Johnson, R. M., Pujol, R., and Puel, J. L. (2003). Salicylate induces tinnitus through activation of cochlear NMDA receptors. J. Neurosci. 23, 3944-3952.

Hakuba, N., Koga, K., Gyo, K., Usami, S. I., and Tanaka, K. (2000). Exacerbation of noise-induced hearing loss in mice lacking the glutamate transporter GLAST. J. Neurosci. 20, 8750-8753.

Hickox, A. E., and Liberman, M. C. (2014). Is noise-induced cochlear neuropathy key to the generation of hyperacusis or tinnitus? J. Neurophysiol. 111, 552-564. doi: $10.1152 /$ jn.00184.2013

Hitchcock, J. M., and Davis, M. (1991). Efferent pathway of the amygdala involved in conditioned fear as measured with the fear-potentiated startle paradigm. Behav. Neurosci. 105, 826-842. doi: 10.1037/0735-7044.105. 6.826

Holt, A. G., Bissig, D., Mirza, N., Rajah, G., and Berkowitz, B. (2010). Evidence of key tinnitus-related brain regions documented by a unique combination of manganese-enhanced MRI and acoustic startle reflex testing. PLoS One 5:e14260. doi: 10.1371/journal.pone.0014260

Ison, J. R., and Bowen, G. P. (2000). Scopolamine reduces sensitivity to auditory gaps in the rat, suggesting a cholinergic contribution to temporal acuity. Hear. Res. 145, 169-176. doi: 10.1016/s0378-5955(00)00088-5

Ison, J. R., Castro, J., Allen, P., Virag, T. M., and Walton, J. P. (2002). The relative detectability for mice of gaps having different ramp durations at their onset and offset boundaries. J. Acoust. Soc. Am. 112, 740-747. doi: 10.1121/1. 1490352

Ison, J. R., McAdam, D. W., and Hammond, G. R. (1973). Latency and amplitude changes in the acoustic startle reflex of the rat produced by variation in auditory prestimulation. Physiol. Behav. 10, 1035-1039. doi: 10.1016/00319384(73)90185-6

Jin, Z. H., Kikuchi, T., Tanaka, K., and Kobayashi, T. (2003). Expression of glutamate transporter GLAST in the developing mouse cochlea. Tohoku J. Exp. Med. 200, 137-144. doi: 10.1620/tjem.200.137

Kalappa, B. I., Brozoski, T. J., Turner, J. G., and Caspary, D. M. (2014). Single unit hyperactivity and bursting in the auditory thalamus of awake rats directly correlates with behavioural evidence of tinnitus. J. Physiol. 592, 5065-5078. doi: 10.1113/jphysiol.2014.278572

Kalappa, B. I., Soh, H., Duignan, K. M., Furuya, T., Edwards, S., Tzingounis, A. V., et al. (2015). Potent KCNQ2/3-specific channel activator suppresses in vivo epileptic activity and prevents the development of tinnitus. J. Neurosci. 35, 8829-8842. doi: 10.1523/JNEUROSCI.5176-14.2015

Karlsson, R. M., Tanaka, K., Saksida, L. M., Bussey, T. J., Heilig, M., and Holmes, A. (2009). Assessment of glutamate transporter GLAST (EAAT1)-deficient mice for phenotypes relevant to the negative and executive/cognitive symptoms of schizophrenia. Neuropsychopharmacology 34, 1578-1589. doi: 10.1038/npp. 2008.215

Li, S., Choi, V., and Tzounopoulos, T. (2013). Pathogenic plasticity of Kv7.2/3 channel activity is essential for the induction of tinnitus. Proc. Natl. Acad. Sci. U S A 110, 9980-9985. doi: 10.1073/pnas.1302770110

Li, S., Kalappa, B. I., and Tzounopoulos, T. (2015). Noise-induced plasticity of KCNQ2/3 and HCN channels underlies vulnerability and resilience to tinnitus. Elife 4:e07242. doi: 10.7554/eLife.07242

Llano, D. A., Turner, J., and Caspary, D. M. (2012). Diminished cortical inhibition in an aging mouse model of chronic tinnitus. J. Neurosci. 32, 16141-16148. doi: 10.1523/JNEUROSCI.2499-12.2012

Longenecker, R. J., and Galazyuk, A. V. (2011). Development of tinnitus in CBA/CaJ mice following sound exposure. J. Assoc. Res. Otolaryngol. 12, 647-658. doi: 10.1007/s10162-011-0276-1

Longenecker, R. J., and Galazyuk, A. V. (2012). Methodological optimization of tinnitus assessment using prepulse inhibition of the acoustic startle reflex. Brain Res. 1485, 54-62. doi: 10.1016/j.brainres.2012.02.067
Longenecker, R. J., Chonko, K. T., Maricich, S. M., and Galazyuk, A. V. (2014). Age effects on tinnitus and hearing loss in CBA/CaJ mice following sound exposure. Springerplus 3:542. doi: 10.1186/2193-1801-3-542

Lowry, E. R., Kruyer, A., Norris, E. H., Cederroth, C. R., and Strickland, S. (2013). The GluK4 kainate receptor subunit regulates memory, mood and excitotoxic neurodegeneration. Neuroscience 235, 215-225. doi: 10.1016/j.neuroscience. 2013.01.029

McFadden, D., Plattsmier, H. S., and Pasanen, E. G. (1984). Aspirin-induced hearing loss as a model of sensorineural hearing loss. Hear. Res. 16, 251-260. doi: 10.1016/0378-5955(84)90114-x

Meltser, I., Cederroth, C. R., Basinou, V., Savelyev, S., Lundkvist, G. S., and Canlon, B. (2014). TrkB-mediated protection against circadian sensitivity to noise trauma in the murine cochlea. Curr. Biol. 24, 658-663. doi: 10.1016/j.cub. 2014.01.047

Middleton, J. W., Kiritani, T., Pedersen, C., Turner, J. G., Shepherd, G. M., and Tzounopoulos, T. (2011). Mice with behavioral evidence of tinnitus exhibit dorsal cochlear nucleus hyperactivity because of decreased GABAergic inhibition. Proc. Natl. Acad. Sci. U S A 108, 7601-7606. doi: 10.1073/pnas. 1100223108

Mongan, E., Kelly, P., Nies, K., Porter, W. W., and Paulus, H. E. (1973). Tinnitus as an indication of therapeutic serum salicylate levels. JAMA 226, 142-145. doi: 10. 1001/jama.1973.03230020014004

Ohlemiller, K. K., and Gagnon, P. M. (2004). Apical-to-basal gradients in age-related cochlear degeneration and their relationship to "primary" loss of cochlear neurons. J Comp Neurol 479, 103-116. doi: 10.1002/cne. 20326

Plappert, C. F., Kuhn, S., Schnitzler, H. U., and Pilz, P. K. (2006). Experience increases the prepulse inhibition of the acoustic startle response in mice. Behav. Neurosci. 120, 16-23. doi: 10.1037/0735-7044.120.1.16

Poirrier, A. L., Van den Ackerveken, P., Kim, T. S., Vandenbosch, R., Nguyen, L., Lefebvre, P. P., et al. (2010). Ototoxic drugs: difference in sensitivity between mice and guinea pigs. Toxicol. Lett. 193, 41-49. doi: 10.1016/j.toxlet.2009.12. 003

Puel, J. L., and Guitton, M. J. (2007). Salicylate-induced tinnitus: molecular mechanisms and modulation by anxiety. Prog. Brain Res. 166, 141-146. doi: 10 1016/s0079-6123(07)66012-9

Ruel, J., Chabbert, C., Nouvian, R., Bendris, R., Eybalin, M., Leger, C. L., et al. (2008). Salicylate enables cochlear arachidonic-acid-sensitive NMDA receptor responses. J. Neurosci. 28, 7313-7323. doi: 10.1523/JNEUROSCI.5335-07.2008

Ruel, J., Wang, J., Rebillard, G., Eybalin, M., Lloyd, R., Pujol, R., et al. (2007). Physiology, pharmacology and plasticity at the inner hair cell synaptic complex. Hear. Res. 227, 19-27. doi: 10.1016/j.heares.2006.08.017

Ruttiger, L., Singer, W., Panford-Walsh, R., Matsumoto, M., Lee, S. C., Zuccotti, A., et al. (2013). The reduced cochlear output and the failure to adapt the central auditory response causes tinnitus in noise exposed rats. PLoS One 8:e57247. doi: 10.1371/journal.pone.0057247

Schmid, A., Koch, M., and Schnitzler, H. U. (1995). Conditioned pleasure attenuates the startle response in rats. Neurobiol. Learn. Mem. 64, 1-3. doi: 10. 1006/nlme.1995.1037

Searchfield, G. D., Munoz, D. J., and Thorne, P. R. (2004). Ensemble spontaneous activity in the guinea-pig cochlear nerve. Hear. Res. 192, 23-35. doi: 10.1016/j. heares.2004.02.006

Shimizu, Y., Hakuba, N., Hyodo, J., Taniguchi, M., and Gyo, K. (2005). Kanamycin ototoxicity in glutamate transporter knockout mice. Neurosci. Lett. 380, 243-246. doi: 10.1016/j.neulet.2005.01.066

Stolzberg, D., Salvi, R. J., and Allman, B. L. (2012). Salicylate toxicity model of tinnitus. Front. Syst. Neurosci. 6:28. doi: 10.3389/fnsys.2012.00028

Sturman, J. A., Dawkins, P. D., McArthur, N., and Smith, M. J. (1968). The distribution of salicylate in mouse tissues after intraperitoneal injection. J. Pharm. Pharmacol. 20, 58-63. doi: 10.1111/j.2042-7158.1968.tb09619.x

Su, Y. Y., Luo, B., Jin, Y., Wu, S. H., Lobarinas, E., Salvi, R. J., et al. (2012). Altered neuronal intrinsic properties and reduced synaptic transmission of the rat's medial geniculate body in salicylate-induced tinnitus. PLoS One 7:e46969. doi: 10.1371/journal.pone.0046969

Sun, W., Lu, J., Stolzberg, D., Gray, L., Deng, A., Lobarinas, E., et al. (2009). Salicylate increases the gain of the central auditory system. Neuroscience 159, 325-334. doi: 10.1016/j.neuroscience.2008.12.024

Turner, J. G., Brozoski, T. J., Bauer, C. A., Parrish, J. L., Myers, K., Hughes, L. F., et al. (2006). Gap detection deficits in rats with tinnitus: a potential 
novel screening tool. Behav. Neurosci. 120, 188-195. doi: 10.1037/0735-7044. 120.1.188

Turner, J. G., and Parrish, J. (2008). Gap detection methods for assessing salicylateinduced tinnitus and hyperacusis in rats. Am. J. Audiol. 17, S185-S192. doi: 10. 1044/1059-0889(2008/08-0006)

Vandesompele, J., De Preter, K., Pattyn, F., Poppe, B., Van Roy, N., De Paepe, A., et al. (2002). Accurate normalization of real-time quantitative RT-PCR data by geometric averaging of multiple internal control genes. Genome Biol. 3:RESEARCH0034. doi: 10.1186/gb2002-3-7-research0034

Vikhe Patil, K., Canlon, B., and Cederroth, C. R. (2015). High quality RNA extraction of the mammalian cochlea for qRT-PCR and transcriptome analyses. Hear. Res. 325, 42-48. doi: 10.1016/j.heares.2015.03.008

von der Behrens, W. (2014). Animal models of subjective tinnitus. Neural Plast. 2014:741452. doi: 10.1155/2014/741452

Wang, H. T., Luo, B., Zhou, K. Q., Xu, T. L., and Chen, L. (2006). Sodium salicylate reduces inhibitory postsynaptic currents in neurons of rat auditory cortex. Hear. Res. 215, 77-83. doi: 10.1016/j.heares.2006. 03.004

Wang, H., Brozoski, T. J., Turner, J. G., Ling, L., Parrish, J. L., Hughes, L. F., et al. (2009). Plasticity at glycinergic synapses in dorsal cochlear nucleus of rats with behavioral evidence of tinnitus. Neuroscience 164, 747-759. doi: 10.1016/j. neuroscience.2009.08.026

Watase, K., Hashimoto, K., Kano, M., Yamada, K., Watanabe, M., Inoue, Y., et al. (1998). Motor discoordination and increased susceptibility to cerebellar injury in GLAST mutant mice. Eur. J. Neurosci. 10, 976-988. doi: 10.1046/j.1460-9568. 1998.00108.x

Weible, A. P., Moore, A. K., Liu, C., DeBlander, L., Wu, H., Kentros, C., et al. (2014). Perceptual gap detection is mediated by gap termination responses in auditory cortex. Curr. Biol. 24, 1447-1455. doi: 10.1016/j.cub.2014.05.031

Willott, J. F., Tanner, L., O'Steen, J., Johnson, K. R., Bogue, M. A., and Gagnon, L. (2003). Acoustic startle and prepulse inhibition in 40 inbred strains of mice. Behav. Neurosci. 117, 716-727. doi: 10.1037/0735-7044.117.4.716

Yi, B., Hu, S., Zuo, C., Jiao, F., Lv, J., Chen, D., et al. (2016). Effects of long-term salicylate administration on synaptic ultrastructure and metabolic activity in the rat CNS. Sci. Rep. 6:24428. doi: 10.1038/srep24428

Zhang, J., Zhang, Y., and Zhang, X. (2011). Auditory cortex electrical stimulation suppresses tinnitus in rats. J. Assoc. Res. Otolaryngol. 12, 185-201. doi: 10. $1007 /$ s10162-010-0246-z

Conflict of Interest Statement: CRC received consulting fees from Sensorion Pharmaceuticals.

Copyright (C) 2016 Yu, Vikhe Patil, Han, Fabella, Canlon, Someya and Cederroth. This is an open-access article distributed under the terms of the Creative Commons Attribution License (CC BY). The use, distribution and reproduction in other forums is permitted, provided the original author(s) or licensor are credited and that the original publication in this journal is cited, in accordance with accepted academic practice. No use, distribution or reproduction is permitted which does not comply with these terms. 